\title{
Phosphine ligands and nitrogen bases in the solvent-free Heck reaction of butenone with aryl iodides. A highly selective synthesis of benzalacetones
}

\author{
Sandro Cacchi,* Giancarlo Fabrizi, and Antonella Goggiamani \\ Dipartimento di Studi di Chimica e Tecnologia delle Sostanze Biologicamente Attive \\ P. le A. Moro 5, 00185 Rome \\ E-mail: sandro.cacchi@uniroma1.it
}

Dedicated to Professor Keiichiro Fukumoto on the occasion of his $70^{\text {th }}$ birthday

(received 20 May 03; accepted 24 June 03; published on the web 25 June 03)

\begin{abstract}
The solvent-free palladium-catalyzed reaction of butenone with aryl iodides has been studied. Phosphine ligands have been found to affect the vinylic substitution to hydroarylation (conjugate addition type) ratio. The nature of the nitrogen base also plays a role in controlling the product selectivity. In the presence of $\mathrm{Pd}(\mathrm{OAc})_{2}$, tris-(2,4,6-trimethoxyphenyl)phosphine and proton sponge the reaction affords exclusively vinylic substitution products usually in high to excellent yield.
\end{abstract}

Keywords: Palladium, Heck reaction, hydroarylation, conjugate addition, benzalacetones

\section{Introduction}

The palladium-catalyzed Heck reaction of $\alpha, \beta$-enones with aryl halides represents a very useful procedure for the preparation of vinylic substitution products. With butenone as the starting olefin, it provides an easy access to benzalacetones 3 , useful reaction intermediates ${ }^{1}$ as well as biologically active compounds. ${ }^{2}$

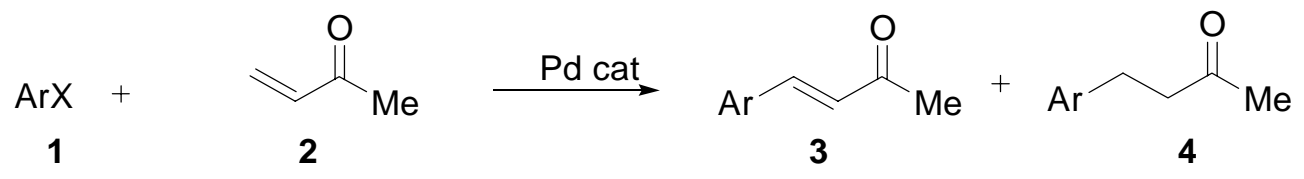

\section{Scheme 1}


However, depending on the reaction conditions, minor amounts of hydroarylation (conjugate addition-type $)^{3}$ derivatives 4 can be formed, making it difficult to isolate pure vinylic substitution products. For example, the reaction of butenone (3 equiv. $)^{4}$ with iodobenzene in the presence of $\mathrm{Pd}(\mathrm{OAc})_{2}, \mathrm{Et}_{3} \mathrm{~N}$ in $\mathrm{DMF}$ at $80{ }^{\circ} \mathrm{C}$ for $24 \mathrm{~h}$ afforded benzalacetone and 4-phenyl-2-butanone in $84 \%$ yield as an approx. 96:4 mixture. The addition of $\mathrm{PPh}_{3}$ led to the isolation of $\beta$-aryl derivatives in 87\% yield, as an approx. 88:12 vinylic substitution ( $v s)$ :hydroarylation $(h)$ mixture. We were intrigued by the observed phosphine effect on the reaction outcome, and found it to be of interest to investigate the reaction further. Particularly, because of our interest in environmentally friendly processes, ${ }^{3 n, 5}$ we decided to investigate the palladium-catalyzed reaction of butenone with aryl iodides under solvent-free conditions. ${ }^{6}$ We now report the results of this study.

\section{Results and Discussion}

We initially attempted the reaction of butenone with $\mathrm{PhI}$ at $80{ }^{\circ} \mathrm{C}$, in the presence of $\mathrm{Et}_{3} \mathrm{~N}$ and catalytic amounts of $\mathrm{Pd}(\mathrm{OAc})_{2}$ in the absence of phosphine ligands. Analysis of the reaction mixture after $14 \mathrm{~h}$ revealed the formation of benzalacetone in 56\% yield along with 4-phenyl-2butanone (18\%). Keeping phenyl iodide as the phenyl donor and $\mathrm{Et}_{3} \mathrm{~N}$ as the base, we then explored the effect of phosphine ligands. As shown in Table 1, phosphine ligands were found to influence the vinylic substitution to hydroarylation ratio. In particular, their presence usually influences the vs: $h$ ratio so as to increase the amount of the hydroarylation product compared the reaction without phosphine ligands (Table 1, entries 2-6).

A possible explanation for the formation of 4 (Scheme 2a; the ligands are not represented for the sake of simplicity) considers the reduction of the carbopalladation adduct 5 by $\mathrm{Et}_{3} \mathrm{~N}$. The ability of $\mathrm{Et}_{3} \mathrm{~N}$ to reduce $\sigma$-organopalladium adducts has already been observed by $\mathrm{us}^{3 \mathrm{a}, \mathrm{n}, 7 \mathrm{c}}$ and others. $^{3 e, 31,7 a, b}$ Stokker $^{3 e}$ showed that $\alpha$-deuterated hydroarylation products could be obtained when a cyclic $\alpha, \beta$-unsaturated lactone was treated with phenyl iodide in the presence of perdeuterated triethylamine and catalytic amounts of $\mathrm{Pd}\left(\mathrm{PPh}_{3}\right)_{4}$. 


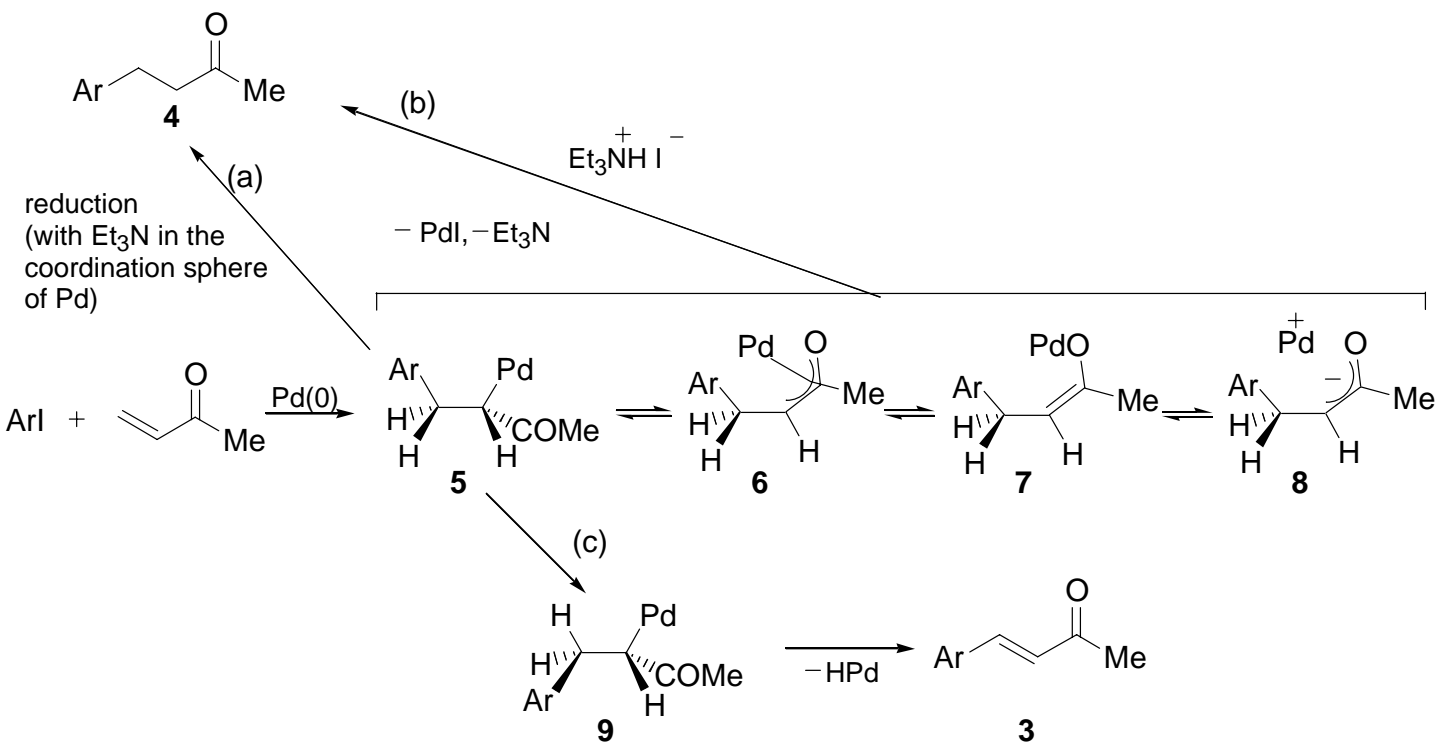

\section{Scheme 2}

The phosphine's effect on the product selectivity and the ability of $\mathrm{Et}_{3} \mathrm{~N}$ to reduce $\sigma$ organopalladium complexes may suggest that both the phosphine ligand and $\mathrm{Et}_{3} \mathrm{~N}$ can coordinate to the palladium atom in the $\sigma$-alkylpalladium intermediate 5 (the latter via displacement of iodide anions or phosphine ligands ${ }^{8}$ ). In the absence of phosphines, i.e., when 5 should contain only $\mathrm{Et}_{3} \mathrm{~N}$ in the coordination sphere of palladium, the vinylic substitution path is envisioned to be favored (Table 1, entry 1) by the low energy requirements for reaching the syn-planar arrangement of the $\mathrm{C}-\mathrm{H}$ bond and the adjoining $\mathrm{C}-\mathrm{Pd}$ bond, as shown in 9 (Scheme 2c). In the presence of phosphines - when the reaction is likely to involve at least one phosphine ligand linked to the palladium atom of 5 - the steric congestion associated with attaining this alignment could increase the energy requirements of the transition state and consequently favor the competing reduction path. For example, with $\mathrm{PPh}_{3}$ the $v$ s: $h$ ratio is 54:46 (Table 1, entry 2).

An alternative, or competing, mechanism (Scheme 2b) for the substitution of the $\mathrm{C}-\mathrm{H}$ bond for the C-Pd bond that does not involve the reduction of the carbopalladation adduct 5 considers the protonation of the anionic fragment of 5. Triethylammonium iodide might be involved in this step. The role of acids in favoring the hydroarylation path has been already observed. ${ }^{3 \mathrm{i}}$ In this case, the palladium carbon-bound enolate complex 5 might be in equilibrium with its synthetic equivalents 6-8. $\mathrm{Et}_{3} \mathrm{~N}$ could act as a reducing agent in the regeneration of $\operatorname{Pd}(0)$ species from the $\mathrm{Pd}(\mathrm{II})$ species derived from the protonation of the $\mathrm{Pd}(\mathrm{II})$-enolate intermediate(s). ${ }^{9}$

Table 1. Palladium-catalyzed reaction of phenyl iodide with butenone ${ }^{\mathrm{a}}$

\begin{tabular}{ccccccc}
\hline Entry & Base & & Catalyst & \multicolumn{2}{c}{ Total } & \multicolumn{2}{c}{ Yield $(\%)$} & $v s: h^{\mathrm{c}}$ \\
& & & yield $(\%)^{\mathrm{b}}$ & $3 \mathrm{a}$ & $4 \mathrm{a}$ & \\
\hline 1 & $\mathrm{Et}_{3} \mathrm{~N}$ & $\mathrm{Pd}(\mathrm{OAc})_{2}$ & 73 & 56 & 18 & $77: 23$
\end{tabular}




\begin{tabular}{|c|c|c|c|c|c|c|}
\hline \multirow[t]{2}{*}{ Entry } & \multirow{2}{*}{ Base } & \multirow[t]{2}{*}{ Catalyst } & \multirow{2}{*}{$\begin{array}{c}\text { Total } \\
\text { yield }(\%)^{b}\end{array}$} & \multicolumn{2}{|c|}{ Yield (\%) } & \multirow[t]{2}{*}{$v s: h^{\mathrm{c}}$} \\
\hline & & & & $3 a$ & $4 a$ & \\
\hline 2 & $\mathrm{Et}_{3} \mathrm{~N}$ & $\mathrm{Pd}(\mathrm{OAc})_{2} / \mathrm{PPh}_{3}$ & 80 & 43 & 37 & $54: 46$ \\
\hline 3 & $\mathrm{Et}_{3} \mathrm{~N}$ & $\mathrm{Pd}\left(\mathrm{PPh}_{3}\right)_{4}$ & 63 & 29 & 34 & $46: 54$ \\
\hline 4 & $\mathrm{Et}_{3} \mathrm{~N}$ & $\mathrm{Pd}(\mathrm{OAc})_{2} / \mathrm{P}(2-\text { tol })_{3}$ & 78 & 42 & 35 & $55: 45$ \\
\hline 5 & $\mathrm{Et}_{3} \mathrm{~N}$ & $\mathrm{Pd}(\mathrm{OAc})_{2} / \mathrm{P}\left(4-\mathrm{MeOC}_{6} \mathrm{H}_{4}\right)_{3}$ & 79 & 47 & 32 & $60: 40$ \\
\hline 6 & $\mathrm{Et}_{3} \mathrm{~N}$ & $\mathrm{Pd}(\mathrm{OAc})_{2} / \mathrm{P}\left(4-\mathrm{Cl}-\mathrm{C}_{6} \mathrm{H}_{4}\right)_{3}$ & 56 & 22 & 34 & $40: 60$ \\
\hline 7 & $\mathrm{Et}_{3} \mathrm{~N}$ & $\mathrm{Pd}(\mathrm{OAc})_{2} / \mathrm{tdmpp}^{\mathrm{d}}$ & 61 & 43 & 18 & $70: 30$ \\
\hline 8 & $\mathrm{Et}_{3} \mathrm{~N}$ & $\mathrm{Pd}(\mathrm{OAc})_{2} / \mathrm{ttmpp}^{\mathrm{e}}$ & 79 & 70 & 9 & $89: 11$ \\
\hline 9 & Proton sponge, & $\mathrm{Pd}(\mathrm{OAc})_{2} / \mathrm{ttmpp}^{\mathrm{e}}$ & 90 & $>89$ & traces & $>99: 1$ \\
\hline 10 & Proton sponge,g & $\mathrm{Pd}(\mathrm{OAc})_{2}$ & 11 & 10 & 1 & $96: 4$ \\
\hline 11 & Proton sponge ${ }^{f, g}$ & $\mathrm{Pd}(\mathrm{OAc})_{2}\left(\mathrm{PPh}_{3}\right)_{2}$ & 30 & 29 & 1 & $97: 3$ \\
\hline
\end{tabular}

${ }^{\text {a }}$ Reactions were carried out at $80^{\circ} \mathrm{C}$ for $14 \mathrm{~h}$, using $0.200 \mathrm{~g}(0.98 \mathrm{mmol})$ of $\mathrm{PhI}$ and the following molar ratios: PhI:butenone:base:Pd:ligand (when added) = 1:3:3:0.05:0.1. ${ }^{\mathrm{b}}$ Calculated on the isolated products. Calculated by ${ }^{1} \mathrm{H}$ NMR analysis of the isolated vs:h mixtures. ${ }^{\mathrm{d}}$ Tris-(2,6-dimethoxyphenyl)-phosphine. 'Tris-(2,4,6-trimethoxyphenyl)phosphine. ${ }^{\mathrm{f}}$ 1,8-bis-(dimethylamino)naphthalene. ${ }^{\mathrm{g}} \mathrm{PhI}$ : proton sponge =1:1.

Whatever the real nature of the reaction intermediates may be, it remains that in the presence of a variety of phosphine ligands the reaction of phenyl iodide with butenone gives a greater proportion of the hydroarylation product than it does when phosphine ligands are omitted. Notably, only upon going to the electron-rich sterically encumbered ligand tris- $(2,4,6$ trimethoxyphenyl)phosphine (ttmpp) does the vinylic substitution product form in yield significantly higher than in the absence of phosphines (Table 1, entry 8).

A working hypothesis accounting for the beneficial effect of ttmpp considers coordinatively unsaturated palladium species ${ }^{10}$ such as 5' (that do not readily proceed along the reduction path) as the main. $\sigma$-alkylpalladium intermediates. Their formation might be favored by the steric congestion around the bonding face of the ligand and by its high basicity. ${ }^{11}$ The electrondonating ability of the ligand could stabilize palladium in its $2^{+}$oxidation state even when the Pd-P bond length presumably increases because of the ligand size.
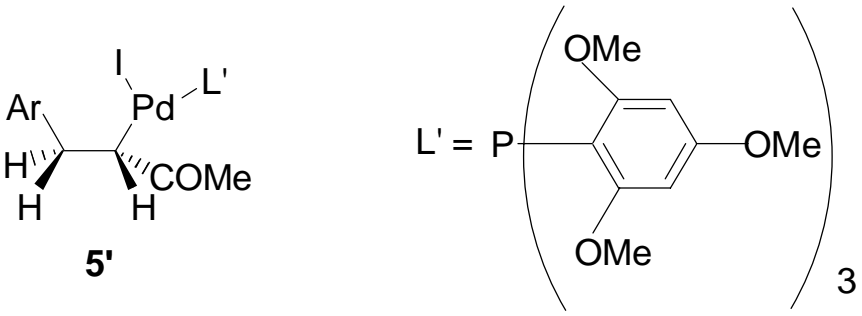

tris(2,4,6-trimethoxyphenyl)phosphine (ttmpp) 
We next turned our attention to the amine base. Surmising that the employment of a more hindered amine could hamper its coordination to palladium and further improve the selectivity for vinylic substitution vs reduction (Scheme 2a), we subjected butenone and phenyl iodide to ttmpp, $\quad \operatorname{Pd}(\mathrm{OAc})_{2}$ and the sterically demanding proton-sponge [1,8-bis(dimethylamino)naphthalene]. We were pleased to find that, under these conditions, 4 a could be isolated as almost the only $\beta$-phenyl product, in excellent yield (Table 1, entry 9). High vinylic substitution to hydroarylation ratios were also obtained when the reaction was run in the presence of proton sponge, but omitting the ligand or substituting $\mathrm{PPh}_{3}$ for ttmpp (Table 1, entries 10 and 11), but the yields were low. Both ttmpp and proton sponge are required to give the best results. Clearly, proton sponge is also a stronger base than triethylamine and this could favor the vinylic substitution path by preventing protonation of palladium-enolate intermediates (Scheme 2b). However, we have not made any attempt to clear this point up.

Using the ttmpp/proton sponge combination, the study was extended to include other aryl iodides. The preparative results are summarized in Table 2.

A number of aryl iodides containing electron-donating and electron-withdrawing substituents gave the corresponding vinylic substitution products in high- to excellent yield. Only 2iodoanisole produced the Heck derivative in low yield (Table 2, entry 5), presumably because of the higher energy requirements for the rotation around the $\mathrm{C}_{\alpha}-\mathrm{C}_{\beta}$ bond of the corresponding carbopalladation adduct (owing to the ortho substituent).

In conclusion, we have demonstrated that under the best conditions developed so far $\left[\mathrm{Pd}(\mathrm{OAc})_{2}\right.$, ttmpp, proton sponge, $\left.80^{\circ} \mathrm{C}\right]$, benzalacetones containing a number of functional groups can be prepared in good to excellent yield from aryl iodides and butenone under solventfree conditions. The observation that phosphine ligands and nitrogen bases can influence the reaction course in this process, and that it is possible to take advantage of their properties to develop a highly selective reaction, suggests an exploration of their employment in other synthetic applications.

Table 2. Palladium-catalyzed reaction of aryl iodides $\mathbf{1}$ with butenone ${ }^{\mathrm{a}}$

\begin{tabular}{clcc}
\hline Entry & Aryl iodide 1 & \multicolumn{2}{c}{ Yield $^{\mathrm{b}}$ of $\mathbf{3}(\%)$} \\
\hline 1 & PhI & $\mathrm{a}$ & 89 \\
2 & $4-\mathrm{Me}-\mathrm{C}_{6} \mathrm{H}_{4}-\mathrm{I}$ & $\mathrm{b}$ & 80 \\
3 & $3-\mathrm{Me}-\mathrm{C}_{6} \mathrm{H}_{4}-\mathrm{I}$ & $\mathrm{c}$ & 67 \\
4 & $4-\mathrm{MeO}-\mathrm{C}_{6} \mathrm{H}_{4}-\mathrm{I}$ & $\mathrm{d}$ & 82 \\
5 & $3-\mathrm{MeO}-\mathrm{C}_{6} \mathrm{H}_{4}-\mathrm{I}$ & $\mathrm{e}$ & 87 \\
6 & $2-\mathrm{MeO}-\mathrm{C}_{6} \mathrm{H}_{4}-\mathrm{I}$ & $\mathrm{f}$ & $32^{\mathrm{c}}$ \\
7 & $4-\mathrm{Me}-3-\mathrm{O}_{2} \mathrm{~N}-\mathrm{C}_{6} \mathrm{H}_{4}-\mathrm{I}$ & $\mathrm{g}$ & 98 \\
8 & $4-\mathrm{Cl}_{6} \mathrm{C}_{6} \mathrm{H}_{4}-\mathrm{I}$ & $\mathrm{h}$ & 98 \\
9 & $3-\mathrm{CF}_{3}-\mathrm{C}_{6} \mathrm{H}_{4}-\mathrm{I}$ & $\mathrm{i}$ & 96 \\
10 & $4-\mathrm{MeCO}_{-} \mathrm{C}_{6} \mathrm{H}_{4}-\mathrm{I}$ & $\mathrm{j}$ & 85 \\
\hline
\end{tabular}


${ }^{\text {a }}$ Reactions were carried out at $80{ }^{\circ} \mathrm{C}$ for $14 \mathrm{~h}$, using 1 equiv. of 1,3 equiv. of butenone, 1.1 equiv. of proton sponge, 0.05 equiv. of $\mathrm{Pd}(\mathrm{OAc})_{2}$, and 0.1 equiv. of ttmpp. ${ }^{\mathrm{b}}$ Yields are given for isolated products. ${ }^{\mathrm{c}}$ 2-Iodoanisole was recovered in $64 \%$ yield.

\section{Experimental Section}

General Procedures. Melting points were determined with a Büchi apparatus and are uncorrected. All of the starting materials, catalyst, ligands, bases, and solvents are commercially available and were used as purchased, without further purification. Reaction products were purified on axially compressed columns (packed with $\mathrm{SiO}_{2}$ 25-40 nm, Macherey Nagel) connected to a Gilson solvent delivery system and to a Gilson refractive index detector. $n$ Hexane/EtOAc mixtures were used for elution. ${ }^{1} \mathrm{H}$ NMR spectra $\left(\mathrm{CDCl}_{3} ; \mathrm{TMS}\right.$ as internal standard) were recorded at $200 \mathrm{MHz} .{ }^{13} \mathrm{C}$ NMR spectra were recorded at $50.3 \mathrm{MHz}$. IR spectra were recorded in $\mathrm{KBr}$ dispersions unless otherwise stated.

\section{Typical procedure for the palladium-catalyzed vinylic substitution of butenone (3g)}

A $5 \mathrm{~mL}$ Supelco vial equipped with a triangular magnetic bar was charged with 4-methyl-3nitrophenyl iodide $(0.263 \mathrm{~g}, 1 \mathrm{mmol})$, butenone $(0.245 \mathrm{~mL}, 3 \mathrm{mmol})$, proton sponge $(0.235 \mathrm{~g}, 1.1$ $\mathrm{mmol}), \mathrm{Pd}(\mathrm{OAc})_{2}(0.011 \mathrm{~g}, 0.05 \mathrm{mmol})$ and ttmpp $(0.053 \mathrm{~g}, 0.10 \mathrm{mmol})$. The vial was sealed and heated at $80{ }^{\circ} \mathrm{C}$ for $14 \mathrm{~h}$. After this time, the reaction mixture was diluted with diethyl ether, the organic layer washed with $0.1 \mathrm{M} \mathrm{HCl}$, saturated $\mathrm{NaHCO}_{3}$, saturated $\mathrm{NaCl}$, dried over $\mathrm{Na}_{2} \mathrm{SO}_{4}$ and concentrated under reduced pressure. The residue was chromatographed on silica gel eluting with a $n$-hexane/EtOAc 85/15 ( $v / v)$ mixture to afford $0.200 \mathrm{~g}(98 \%$ yield) of $3 \mathbf{g}$ as a yellow solid: mp 87-88 ${ }^{\circ} \mathrm{C}$; IR 1674, 1525, 1348, 979, $819 \mathrm{~cm}^{-1} ;{ }^{1} \mathrm{H}$ NMR $\delta 8.04$ (d, J=1.5 Hz, 1H), 7.59 (dd, $\mathrm{J}=8.2 \mathrm{~Hz}, J=1.5 \mathrm{~Hz}, 1 \mathrm{H}), 7.41(\mathrm{~d}, J=16.2 \mathrm{~Hz}, 1 \mathrm{H}), 7.31(\mathrm{~d}, J=8.2 \mathrm{~Hz}, 1 \mathrm{H}), 6.68(\mathrm{~d}, J=16.2$ $\mathrm{Hz}, 1 \mathrm{H}), 2.53$ (s, 3H), 2.32 (s, 3H); ${ }^{13} \mathrm{C}$ NMR $\delta 197.8,149.4,140.3,135.5,133.7,133.5,131.9$, 128.5, 124.0, 27.8, 20.5; MS m/e (relative intensity) $205\left(\mathrm{M}^{+}, 26\right), 190$ (100), 160 (11), 144 (24), 143 (27). Anal. Calcd for $\mathrm{C}_{11} \mathrm{H}_{11} \mathrm{NO}_{3}$ : C, 64.37; H, 5.41; N, 6.83. Found: C, 64.43; H, 5.42; N, $6.85 \%$.

3a. (pale yellow solid) mp 38-39; lit. ${ }^{13} \mathrm{mp} 36-39{ }^{\circ} \mathrm{C}$.

3b. pale yellow oil; IR (Nujol) 1669, 1257, $803 \mathrm{~cm}^{-1}$; ${ }^{1} \mathrm{H}$ NMR $\delta 7.48(\mathrm{~d}, J=16.2 \mathrm{~Hz}, 1 \mathrm{H}), 7.46$ $(\mathrm{d}, J=8.2 \mathrm{~Hz}, 2 \mathrm{H}), 7.22$ (d, $J=8.2 \mathrm{~Hz}, 2 \mathrm{H}), 6.70(\mathrm{~d}, J=16.2 \mathrm{~Hz}, 1 \mathrm{H}), 2.39(\mathrm{~s}, 6 \mathrm{H}) ;{ }^{13} \mathrm{C} \mathrm{NMR}$ б. 198.4, 143.4, 140.9, 131.4, 129.5, 128.1, 126.0, 27.2, 21.3; MS m/e (relative intensity) 160 $\left(\mathrm{M}^{+}, 22\right), 145$ (100), 117 (50). Anal. Calcd for $\mathrm{C}_{11} \mathrm{H}_{12} \mathrm{O}: \mathrm{C}, 82.45$; H, 7.55. Found: C, 82.53; H, $7.54 \%$.

3c. pale yellow oil; IR (Nujol) 1672, 1257, 778, $691 \mathrm{~cm}^{-1} ;{ }^{1} \mathrm{H}$ NMR $\delta .7 .40$ (d, J =16.2 Hz, 1H), 7.28-7.02 (m, 4H), 6.59 (d, J = 16.2 Hz, 1H), $2.55(\mathrm{~s}, 6 \mathrm{H}) ;{ }^{13} \mathrm{C}$ NMR $\oint 198.3,143.6,138.5$, 134.2, 131.3, 128.8, 128.7, 126.8, 125.4, 27.3, 21.2; MS m/e (relative intensity) $160\left(\mathrm{M}^{+}, 7\right), 145$ (100), 115 (44). Anal. Calcd for $\mathrm{C}_{11} \mathrm{H}_{12} \mathrm{O}$ : C, 82.45; H, 7.55. Found: C, 82.38; H, 7.56\%. 
3d. (pale yellow solid) $\mathrm{mp} 68-69^{\circ} \mathrm{C}$; lit. $^{13} \mathrm{mp} 70-72{ }^{\circ} \mathrm{C}$.

3e. pale yellow oil; lit. ${ }^{14}$ bp $110-115^{\circ} \mathrm{C}$ (0.030 Torr); IR (Nujol) 1673, 125, 782, 732, $689 \mathrm{~cm}^{-1}$; ${ }^{1} \mathrm{H}$ NMR $\delta 7.45(\mathrm{~d}, J=16.3 \mathrm{~Hz}, 1 \mathrm{H}), 7.29(\mathrm{t}, J=7.8 \mathrm{~Hz}, 1 \mathrm{H}), 7.16-7.00(\mathrm{~m}, 2 \mathrm{H}), 6.93(\mathrm{dt}, J=$ $8.2 \mathrm{~Hz}, J=0.7 \mathrm{~Hz}, 1 \mathrm{H}), 6.67(\mathrm{~d}, J=16.3 \mathrm{~Hz}, 1 \mathrm{H}), 3.80(\mathrm{~s}, 3 \mathrm{H}), 2.36(\mathrm{~s}, 3 \mathrm{H}) ;{ }^{13} \mathrm{C}$ NMR $\delta 198.3$, $159.7,143.2,135.5,129.7,127.1,120.8,116.1,112.8,55.0,27.2$; MS $\mathrm{m} / \mathrm{e}$ (relative intensity) $176\left(\mathrm{M}^{+}, 64\right), 161$ (100), 133 (52), 118 (50). Anal. Calcd for $\mathrm{C}_{11} \mathrm{H}_{12} \mathrm{O}_{2}: \mathrm{C}, 74.96 ; \mathrm{H}, 6.87$. Found: C, $75.04 ; \mathrm{H}, 6.88 \%$.

3f. (pale yellow solid) mp $45-46{ }^{\circ} \mathrm{C}$; IR $1669,1248,754 \mathrm{~cm}^{-1} ;{ }^{1} \mathrm{H}$ NMR $\delta 7.88(\mathrm{~d}, J=16.5 \mathrm{~Hz}, 1$ H), 7.52 (dd, $J=7.6 \mathrm{~Hz}, J=1.5 \mathrm{~Hz}, 1 \mathrm{H}), 7.35$ (td, $J=7.0 \mathrm{~Hz}, J=1.5 \mathrm{~Hz}, 1 \mathrm{H}), 7.01-6.82$ (m, 2 $\mathrm{H}), 6.74(\mathrm{~d}, J=16.5 \mathrm{~Hz}, 1 \mathrm{H}), 3.87(\mathrm{~s}, 3 \mathrm{H}), 2.38(\mathrm{~s}, 3 \mathrm{H}) ;{ }^{13} \mathrm{C}$ NMR $\delta$ 199.2, 158.2, 138.8, $131.9,128.3,127.7,123.2,120.8,111.1,55.5,27.1 ; \mathrm{MS} m / e$ (relative intensity) $176\left(\mathrm{M}^{+}, 13\right)$, 161 (55), 145 (100), 118 (27). Anal. Calcd for $\mathrm{C}_{11} \mathrm{H}_{12} \mathrm{O}_{2}: \mathrm{C}, 74.96 ; \mathrm{H}, 6.87$. Found: C, 74.88; H, $6.86 \%$.

3h. (pale yellow solid) mp $54-55^{\circ} \mathrm{C}$; lit. ${ }^{13} \mathrm{mp} 54-55^{\circ} \mathrm{C}$.

3i. pale yellow oil; lit. ${ }^{15}$ b.p. $90-92{ }^{\circ} \mathrm{C}(0.2$ Torr $)$; IR $1676,1334,799,697 \mathrm{~cm}^{-1} ;{ }^{1} \mathrm{H}$ NMR $\delta 7.82$ $(\mathrm{m}, 5 \mathrm{H}), 6.75(\mathrm{~d}, J=16.2 \mathrm{~Hz}, 1 \mathrm{H}), 2.38(\mathrm{~s}, 3 \mathrm{H}) ;{ }^{13} \mathrm{C} \mathrm{NMR} \delta 197.8,141.2,135.2,131.2(\mathrm{q}, J=$ $32.4 \mathrm{~Hz}), 131.1,129.4,128.4,126.6(\mathrm{q}, J=3.6 \mathrm{~Hz}), 124.6(\mathrm{q}, J=3.9 \mathrm{~Hz}), 123.7(\mathrm{q}, J=271.0$ $\mathrm{Hz}), 27.5$; MS m/e (relative intensity) $214\left(\mathrm{M}^{+}, 20\right), 199$ (81), 171 (35), 161 (100), 145 (40). Anal. Calcd for $\mathrm{C}_{11} \mathrm{H}_{9} \mathrm{~F}_{3} \mathrm{O}: \mathrm{C}, 61.68 ; \mathrm{H}, 4.24$. Found: $\mathrm{C}, 61.74 ; \mathrm{H}, 4.25 \%$.

3j. (pale yellow solid) $\mathrm{mp} 105-106{ }^{\circ} \mathrm{C}$; IR 1678, 1660, 1263, $817 \mathrm{~cm}^{-1} ;{ }^{1} \mathrm{H}$ NMR $\delta 7.88(\mathrm{~d}, J$ $=8.3 \mathrm{~Hz}, 2 \mathrm{H}), 7.53(\mathrm{~d}, J=8.3 \mathrm{~Hz}, 2 \mathrm{H}), 7.44(\mathrm{~d}, J=16.2 \mathrm{~Hz}, 1 \mathrm{H}), 6.69(\mathrm{~d}, J=16.2 \mathrm{~Hz}, 1 \mathrm{H})$, $2.52(\mathrm{~s}, 3 \mathrm{H}), 2.31(\mathrm{~s}, 3 \mathrm{H}) ;{ }^{13} \mathrm{C}$ NMR $\delta 197.9,197.1,141.5,138.6,137.9,128.9,128.7,128.2$, 27.6, 26.6a; MS m/e (relative intensity) $188\left(\mathrm{M}^{+}, 100\right), 173$ (73), 145 (86), 131 (48), 115 (28). Anal. Calcd for $\mathrm{C}_{12} \mathrm{H}_{12} \mathrm{O}_{2}$ : C, 76.56; H, 6.43. Found: C, $76.64 \mathrm{H}, 6.42 \%$.

\section{Acknowledgments}

Work carried out in the framework of the National Project "Stereoselezione in Sintesi Organica. Metodologie ed Applicazioni" supported by the Ministero dell'Università e della Ricerca Scientifica e Tecnologica, Rome, and by the University "La Sapienza", Rome.

\section{References and Notes}

1. For recent references, see: (a) Alexakis, A.; March, S. J. Org. Chem. 2002, 67, 8753. (b) Park, M. Y.; Yang, S. G.; Kim, Y. H. Heteroatom Chem. 2002, 13, 431. (c) Andriamialisoa, Z.; Valla, A.; Cartier, D.; Labia, R. Helv. Chim. Acta 2002, 85, 2926. (d) Shen, Y.; Feng, X.; Zhang, G.; Jiang, Y. Synlett 2002, 1353. (e) Deng, Guisheng; T., X.; Qu, Z.; Wang, J. Angew. Chem., Internat. Ed. 2002, 41, 2773. (f) Mizutani, H.; Degrado, S. J.; Hoveyda, A. 
H. J. Am. Chem. Soc. 2002, 124, 779. (g) Oi, S.; Honma, Y.; Inoue, Y. Org. Lett. 2002, 4, 667. (h) Wang, Z.; Fetterly, B.; Verkade, J. G. J. Organomet. Chem. 2002, 646, 161.

2. See, for example: (a) Yamagami C.; Motohashi N.; Akamatsu M. Bioorg. Med. Chem. Lett. 2002, 12, 2281. (b) Yamagami, C.; Kishida, N.; Ka, S.; Horiuchi, M.; Motohashi, N. Chem. Pharm. Bull. 1998, 46, 274. (c) Motohashi, N.; Yamagami, C.; Tokuda, H.; Okuda, Y.; Ichiishi, E.; Mukainaka, T.; Nishino, H.; Saito, Y. Mut. Res. 2000, 464, 247. (d) Motohashi, N.; Ashihara, Y.; Yamagami, C.; Saito, Y. Mut. Res. 2001, 474, 113. (e) Yamagami, C.; Motohashi, N. Eur. J. Med. Chem. 2002, 37, 127.

3. (a) Cacchi, S.; Arcadi, A. J. Org. Chem. 1983, 48, 4236. (b) Cacchi, S.; F. La Torre, G. Palmieri J. Organomet. Chem. 1984, 268, C48. (c) Cacchi, S.; G. Palmieri Synlett 1984, 575. (d) Arcadi, A.; Marinelli, F.; Cacchi, S. J. Organomet. Chem. 1986, 312, C27. (e) Stokker, G. E. Tetrahedron Lett. 1987, 28, 3179. (f) Wada, A.; Yasuda, H.; Kanatomo, S. Synthesis 1988, 771. (g) Wolff, S.; Hoffmann, H. M. R. Synthesis 1988 760. (h) Hoffmann, M. H. R.; Schmidt, B.; Wolff, S. Tetrahedron 1989, 45, 6113. (i) Amorese, A.; Arcadi, A.; Bernocchi, E.; Cacchi, S.; Cerrini, S.; Fedeli, W.; Ortar, G. Tetrahedron 1989, 45, 813. (j) Konopelski, J. P.; Chu, K. S.; Negrete, G. R. J. Org. Chem. 1991, 56, 1355. (k) Wada, A.; Ohki, K.; Nagai, S.; Kanatomo, S. J. Heterocyclic Chem. 1991, 28, 509. (l) Friestad, G. K.; Braunchaud, B. P. Tetrahedron Lett. 1995, 36, 7047. (m) Hagiwara, H.; Eda, Y.; Morohashi, K.; Suzuki, T.; Ando, M.; Ito, N. Tetrahedron Lett. 1998, 39, 4055. (n) Cacchi, S.; Fabrizi, G., Gasparrini, F.; Pace, P.; Villani, C. Synlett 2000, 650.

4. Decreasing the excess of butenone leads to the formation of significant amounts of 3,3diphenylbutan-2-one, most probably derived from the palladium-catalyzed hydroarylation of the vinylic substitution product 3a formed initially. For example, subjecting phenyl iodide to 1.5 equiv. of butenone in the presence of $\mathrm{Pd}\left(\mathrm{PPh}_{3}\right)_{4}$ under our usual conditions produced 3a, 4a and 3,3-diphenyl-2-butanone in 13, 21 and 30\% yield, respectively.

5. (a) Cacchi, S.; G. Fabrizi, Gasparrini, F.; Pace, P.; Villani, C. Synlett 1999, 345. (b) Battistuzzi, G.; Cacchi, S.; Fabrizi, G. Synlett 2002, 439. (c) Cacchi, S.; Fabrizi, G.; Goggiamani, A.; Moreno-Manãs, Vallribera, A. Tetrahedron Lett. 2002, 43, 5537.

6. For reviews on solvent-free reactions, see: (a) Dittmer, D. C. Chem. Ind. 1997, 779. (b) Metzger, J. O. Angew. Chem., Internat. Ed. 1998, 37, 2975. (c) Loupy, A.; Petit, Alain; H., J.; Texier-Boullet, F.; Jacquault, P.; Mathe, D. Synthesis 1998, 1213. (d) Tanaka, K.; Toda, F. Chem. Rev. 2000, 100, 1025. (e) Diaz-Ortiz, A.; de la Hoz, A.; Langa, F. Green Chem. 2000, 2, 165. (f) Varma, R. S. Pure Appl. Chem. 2001, 73, 193.

7. (a) Cabri, W.; Candiani, I.; De Bernardis, S.; Francalanci, F.; Penco, S. J. Org. Chem. 1991, 56, 5796. (b) Laschat, S.; Narjes, F.; Overman, L.E. Tetrahedron 1994, 50, 347. (c) Arcadi, A.; Cacchi, S.; Fabrizi, G.; Marinelli, F.; Pace, P. Tetrahedron 1996, 52, 6983.

8. For some examples of phosphine displacement by amines in palladium complexes, see: (a) Widenhoefer, R. A.; Buchwald, S. L. Organometallics 1996, 15, 3534. (b) Khong, H. A.; Widenhoefer, R. A. Organometallics 1997, 36, 2610. 
9. For some leading references on palladium enolates, see: (a) Ito, Y.; Hirao, T.; Saegusa, T. J. Org. Chem. 1978, 43, 1011. (b) Minami, I.; Nisar, M.; Yuhara, M.; Shimizu, I.; Tsuji, J. Synthesis 1987, 992. (c) Nokami, J.; Mandai, T.; Watanabe, H.; Ohyama, H.; Tsuji, J. J. Am. Chem. Soc. 1989, 111, 4126. (d) Burkhardt, E. R.; Bergman, R. G.; Heathcock, C. H. Organometallics 1990, 9, 30. (e) Ogoshi, S.; Morimoto, T.; Nishio, K.-I.; Ohe, K.; Murai, S. J. Org. Chem. 1993, 58, 9. (f) Soeoka, M.; Tokunoh, R.; Miyazaki, F.; Hagiwara, E.; Shibasaki, M. Synlett 1997, 463. (g) Garg, N.; Larhed, M.; Hallberg, A. J. Org. Chem. 1998, 63, 4158. (h) Shim, J.-G.; Nakamura, H.; Yamamoto, Y. J. Org. Chem. 1998, 63, 8470. (i) Kawatsura, M.; Hartwig, J. F. J. Am. Chem. Soc. 1999, 121, 1473. (j) Albéniz, A. C.; Catalina, N. M.; Espinet, P.; Redón, R. Organometallics 1999, 18, 5571. (k) Lei, A.; Zhang, X. Tetrahedron Lett. 2002, 43, 2525.

10. A sterically demanding ligand such as $\mathrm{P}\left(2-\mathrm{tol}_{3}\right)_{3}$ has been reported to create coordinatively unsaturated palladium species: Paul, F.; Patt, J.; Hartwig, J.F. Organometallics 1995, 14, 3030 .

11. Wada, M.; Higashizaki, S. J. Chem. Soc. Chem. Comm. 1984, 482.

12. Tolman, C.A. Chem. Rev. 1977, 77, 313.

13. Elias, G.; Rao, M. Eur. J. Med. Chem. 1988, 23, 379.

14. Evans, D. A.; Gauchet-Prunet, J. A.; Carreira, E. M.; Charette, A. B. J. Org. Chem. 1991, 56, 741 .

15. Edwards, M. L.; Ritter, H. W.; Stemerick, D. M.; Stewart, K. T. J. Med. Chem. 1983, 26, 431. 